\title{
Cárie radicular entre indivíduos em situação de rua: prevalência e fatores associados
} Root caries among homeless people: prevalence and associated factors Caries de raíz en personas sin hogar: prevalencia y factores associados

Maria do Carmo Matias FREIRE Programa de Pós-Graduação em Odontologia, Faculdade de Odontologia, Universidade Federal de Goiás (PPGO-FO-UFG), Goiânia - GO, Brasil https://orcid.org/0000-0001-6078-6728 Juliana Aparecida de Campos LAWDER Programa de Pós-Graduação em Odontologia, Faculdade de Odontologia, Universidade Federal de Goiás (PPGO-FO-UFG), Goiânia - GO, Brasil https://orcid.org/0000-0003-0412-218X João Batista de SOUZA Programa de Pós-Graduação em Odontologia, Faculdade de Odontologia, Universidade Federal de Goiás (PPGO-FO-UFG), Goiânia - GO, Brasil https://orcid.org/0000-0002-7476-9950 Marcos André de MATOS

Faculdade de Enfermagem, Universidade Federal de Goiás (FEN-UFG), Goiânia - GO, Brasil https://orcid.org/0000-0001-8643-7032

\section{Resumo}

Introdução: A cárie radicular tem sido investigada na população em geral, mas a evidência em pessoas em situação de rua é limitada. Objetivo: Investigar a prevalência de cárie radicular e associação com fatores sociodemográficos, uso de drogas e saúde bucal em indivíduos adultos em situação de rua. Material e Método: Estudo transversal com 116 indivíduos de 18 a 68 anos de Goiânia, Goiás. Os dados foram coletados por meio de entrevista e exame clínico, com base nos critérios da Organização Mundial de Saúde. O desfecho foi a prevalência de cárie na raiz. As covariáveis foram as características sociodemográficas, uso de drogas lícitas e ilícitas, número de dentes remanescentes, índice CPOD e uso de prótese parcial removível. $\mathrm{Na}$ análise estatística foram utilizadas comparações bivariadas e regressão de Poisson com variância robusta. Resultados: A prevalência de cárie radicular foi 26,4\% (IC 95\%=19,0-35,5), com média de 0,76 raízes por indivíduo. Todas as raízes afetadas estavam cariadas e não tratadas e os dentes mais afetados foram os posteriores. Maior prevalência foi encontrada em indivíduos com idade, número de dentes remanescentes e índice CPO-D mais elevados. Após ajuste, apenas a idade permaneceu associada ao desfecho $(R P=1,03 ; \mathrm{IC} 95 \%=1,00-1,06)$. Conclusão: Mais de $1 / 4$ dos indivíduos em situação de rua pesquisados apresentaram cárie radicular e a prevalência foi associada ao aumento da idade. A inexistência de raízes restauradas sugere a necessidade de promover o acesso deste grupo populacional aos serviços de saúde.

Descritores: Cárie Radicular; Pessoas em Situação de Rua; Adulto; Idoso; Saúde Bucal; Epidemiologia; Vulnerabilidade em Saúde.

\section{Abstract}

Introduction: Root caries has been investigated in the general population, but the evidence in homeless people is limited. Objective: To investigate the prevalence of root caries and its association with sociodemographic factors, drug use and oral health in homeless persons. Material and Method: Cross-sectional study with 116 individuals aged 18 to 68 years from Goiânia, Goiás. Data were collected through interviews and clinical examination, based on the criteria of the World Health Organization. The outcome was the prevalence of root caries. The covariables were sociodemographic characteristics, use of licit and illicit drugs, number of remaining teeth, DMFT index and use of removable partial denture. In the statistical analysis, bivariate comparisons and Poisson regression with robust variance were used. Results: The prevalence of root caries was $26.4 \%(95 \%$ $\mathrm{Cl}=19.0-35.5)$, with an average of 0.76 roots per individual. All affected roots were decayed and untreated and the most affected teeth were the posterior ones. A higher prevalence was found in individuals with higher age, number of remaining teeth and DMFT index. After adjustment, only age remained associated with the outcome $(\mathrm{PR}=1.03 ; 95 \% \mathrm{Cl}=1.00-1.06)$. Conclusion: More than $1 / 4$ of the homeless individuals surveyed had root caries and the prevalence was associated with increasing age. The lack of restored roots suggests the need to promote the access of this population group to health services.

Descriptors: Root Caries; Homeless Persons; Adults; Elderly; Oral Health; Epidemiology; Health Vulnerability.

\section{Resumen}

Introducción: La caries de raíz se hay investigado en la población general, pero la evidencia en personas sin hogar es limitada. Objetivo: Investigar la prevalencia de caries radicular y su asociación con factores sociodemográficos, consumo de drogas y salud bucal en adultos de la calle. Material y Método: Estudio transversal con 116 individuos de 18 a 68 años de Goiânia, Goiás. Los datos fueron recolectados a través de entrevistas y examen clínico, de acuerdo con los criterios de la Organización Mundial de la Salud. El resultado fue la prevalencia de caries radicular. Las covariables fueron características sociodemográficas, uso de drogas lícitas e ilícitas, número de dientes remanentes, índice CPOD y uso de prótesis parcial removible. En el análisis estadístico se utilizaron comparaciones bivariadas y regresión de Poisson con varianza robusta. Resultados: La prevalencia de caries radicular fue de 26,4\% (IC 95\% = 19,0-35,5), con un promedio de 0,76 raíces por individuo. Todas las raíces afectadas estaban deterioradas y sin tratamiento y los dientes más afectados fueron los posteriores. Se encontró una mayor prevalencia en individuos con mayor edad, número de dientes remanentes e índice CPOD. Después del ajuste, solo la edad permaneció asociada con el resultado $(R P=1,03$; IC del $95 \%=1,00-1,06)$. Conclusión: más de 1/4 de las personas sin hogar encuestadas tenían caries de raíz y la prevalencia se asoció con el aumento de la edad. La falta de raíces restauradas sugiere la necesidad de promover el acceso de este grupo de población a los servicios de salud.

Descriptores: Caries Radicular; Personas sin Hogar; Adulto; Anciano; Salud Bucal; Epidemiología; Vulnerabilidad en Salud. INTRODUÇÃO

A população em situação de rua (PSR) é um dos mais relevantes problemas sociais em muitos países e constitui um reflexo da exclusão social. No Brasil, considera-se PSR "o grupo populacional heterogêneo que possui em comum a pobreza extrema, os vínculos familiares interrompidos ou fragilizados e a inexistência de moradia convencional regular, e que utiliza os logradouros públicos e as áreas degradadas como espaço de moradia e de 
sustento, de forma temporária ou permanente, bem como as unidades de acolhimento para pernoite temporário ou como moradia provisória"1.

Não existem estatísticas oficiais atualizadas sobre esta população no país. Dados obtidos em amostras de municípios que participaram da única Pesquisa Nacional sobre a PSR realizado em $2007-2008^{2}$ e de uma estimativa em $2015^{3}$ mostraram que é nos municípios maiores que se concentra esta população. Supõe-se que o número de pessoas em situação de rua vem crescendo nos últimos anos. Em 2009 foi instituída a primeira Política Nacional para a $\mathrm{PSR}^{1}$ e estratégias de atenção à saúde dirigidas a este grupo foram implementadas ${ }^{4,5}$.

A PSR é uma população vulnerável com dificuldades de acesso aos serviços e geralmente não é incluída em inquéritos populacionais de saúde. Um dos problemas de saúde bucal que pode afetar este grupo populacional é a cárie radicular, causada pelo consumo frequente de açúcares em presença de biofilme dental, e geralmente associada à idade, baixa condição socioeconômica, uso de próteses e outros fatores clínicos, xerostomia, tabagismo e consumo de álcool, dentre outros fatores ${ }^{6,7}$.

Em estudos epidemiológicos na população em geral, a cárie radicular tem sido menos investigada do que a cárie de coroa, embora o interesse tenha sido crescente nas últimas décadas, devido ao aumento da longevidade e do número de dentes naturais remanescentes. Uma revisão sistemática da literatura identificou prevalência global em adultos de $41,5 \%^{8}$. No Brasil, $16,7 \%$ dos adultos e $13,6 \%$ nos idosos apresentavam esta condição em $2010^{9}$. Contudo, apenas dois estudos em cidades da Inglaterra relataram dados sobre a cárie radicular em PSR, com pouca abrangência e sem considerar os aspectos associados à doença ${ }^{10,11}$.

A evidência disponível revela lacunas que incluem a necessidade de descrever a prevalência, bem como explorar os fatores associados à cárie radicular. Considerando que a condição de saúde bucal pode ser influenciada por aspectos socioeconômicos e culturais mais amplos, há ainda a necessidade de estudos em PSR de países em desenvolvimento. Assim, o presente estudo buscou explorar estas questões em PSR de uma capital brasileira e contribuir para ampliar o conhecimento acerca da temática para o embasamento das estratégias de enfrentamento dos problemas de saúde bucal junto a este grupo. O objetivo foi investigar a prevalência de cárie radicular e fatores associados em indivíduos adultos em situação de rua.

MATERIAL E MÉTODO

Foi realizado um estudo transversal, utilizando dados de um projeto mais amplo sobre as condições de saúde de indivíduos em situação de rua, coordenado pela Faculdade de Enfermagem da Universidade Federal de Goiás (UFG).

A amostra de conveniência foi composta por indivíduos frequentando a única instituição pública municipal que abrigava PSR temporariamente no período do estudo, em Goiânia, Goiás, localizada na Região CentroOeste do Brasil. O município possui água de abastecimento fluoretada desde os anos 1980 e a instituição pesquisada não oferecia ações de assistência à saúde. A amostra de conveniência incluiu os indivíduos com idade igual ou superior a 18 anos que estavam na referida instituição no período da coleta dos dados $(\mathrm{N}=835)$. $\mathrm{O}$ projeto mais amplo incluiu uma variedade de indicadores e instrumentos de coleta de dados, que constituíram subprojetos. Para a investigação clínica da saúde bucal, foram convidados 120 indivíduos e 116 aceitaram participar (taxa de resposta $=96,7 \%$ ).

Os dados foram coletados por meio de exame clínico bucal e entrevista, utilizando instrumentos baseados na Pesquisa Nacional de Saúde Bucal - SBBrasil 2010 ${ }^{12}$. O exame clínico foi realizado por uma cirurgiã-dentista, que utilizou critérios adaptados da Organização Mundial da Saúde $(\mathrm{OMS})^{13}$. As variáveis clínicas analisadas no presente estudo foram: prevalência de dentes com cárie radicular, número de dentes remanescentes (presentes no momento do exame), prevalência de dentes com cárie na coroa e uso de prótese parcial removível (sim/não). Foram examinados os 32 dentes de cada indivíduo, utilizando espelho bucal e sonda periodontal recomendada pela OMS. Dentes que não puderam ser examinados devido a condições como bandas ortodônticas ou hipoplasias gengivais severas, foram excluídos.

O desfecho foi a prevalência de cárie radicular (sim/não), que expressa a proporção de indivíduos com um ou mais dentes com experiência de cárie na raiz. Para o diagnóstico de cárie nas raízes dentárias foram registradas as seguintes condições quando 0 dente apresentava raiz exposta (com recessão gengival além da junção amelodentinária): raiz cariada; raiz restaurada, mas cariada; raiz restaurada e sem cárie; e apoio de ponte ou 
coroa. Apenas um registro foi realizado para raiz em cada dente. Dentes com raiz exposta e hígida (sem evidência de cárie ou restauração) não foram registrados. Dentes perdidos por cárie ou outra razão não receberam diagnóstico específico para raiz.

O diagnóstico de cárie nas coroas dentárias foi com base no índice de dentes cariados, perdidos e obturados (CPO-D), sendo registradas as seguintes condições: coroa cariada (cavidade evidente ou restauração temporária); restaurada, mas cariada; restaurada e sem cárie; e dente perdido devido à cárie.

Previamente à coleta dos dados, foi realizada calibração intraexaminadora com um grupo de 10 adultos fora da instituição estudada. Os resultados indicaram boa concordância em quatro dentes para cárie de raiz: 18 (Kappa= 0,82), 21, 37 e 28 (Kappa= $0,80)$; e concordância perfeita (Kappa $=1,0)$ nos demais dentes. Para cárie de coroa, os resultados foram: concordância moderada no dente 13 (Kappa=0,75), boa nos dentes 16 e 37 $($ Kappa $=0,82)$, e $18 \quad($ Kappa $=0,84)$, e concordância perfeita $(K a p p a=1,0)$ nos demais dentes.

O instrumento utilizado na entrevista foi um questionário estruturado com questões fechadas e semiabertas acerca de fatores relacionados à saúde geral e bucal, além de características sociodemográficas. $\mathrm{O}$ questionário foi testado em um estudo piloto com um grupo de 20 indivíduos na própria instituição. As entrevistas foram realizadas em uma sala da própria instituição, destinada exclusivamente à coleta de dados, por uma equipe de estudantes e profissionais da área da saúde, que foram previamente treinados. Após a coleta dos dados, os participantes receberam orientações sobre o autocuidado e os serviços públicos de saúde disponíveis.

A seleção das covariáveis foi com base em estudos anteriores na população em geral: características sociodemográficas, uso de drogas e aspectos relacionados à saúde bucal. As características sociodemográficas foram: idade, sexo (feminino/masculino), escolaridade com base em anos de estudo (alta: 9 anos ou mais/baixa: até 8 anos), estado civil (casado ou união estável/solteiro, separado, divorciado ou viúvo), recebe renda mensal (sim/não) e tempo em situação de rua (até 1 ano/mais de 1 ano). A classificação da cor da pele/raça foi de acordo com as categorias do Instituto Brasileiro de Geografia e Estatística ${ }^{14}$ : brancos, pretos, pardos, amarelos e indígenas. As variáveis relacionadas à saúde bucal foram: frequência diária de higiene bucal (2 vezes ou mais/1 vez ou menos) e tempo desde a última consulta ao dentista (até 2 anos/3 anos ou mais). O uso de drogas lícitas e ilícitas (sim/não) incluiu o consumo diário de bebida alcoólica, uso atual de cigarro, as substâncias ilícitas alguma vez na vida e nos últimos seis meses.

A análise estatística dos dados foi feita no Programa Statistical Package for Social Sciences (SPSS for Windows, versão 21). Inicialmente foi realizada análise descritiva das variáveis e as seguintes estimativas: prevalência de cárie radicular (total de raízes cariadas e/ou restauradas > 1) por indivíduo; médias de dentes com raízes afetadas por indivíduo; e prevalência por dente afetado em relação ao total de dentes remanescentes (dentes presentes no momento do exame). Em seguida foram feitas comparações bivariadas para identificar as covariáveis associadas ao desfecho prevalência de cárie radicular, por meio dos testes Qui-quadrado de Pearson para as variáveis categóricas e Mann-Whitney para as numéricas assimétricas (idade, número de dentes remanescentes e índice CPO-D). As variáveis demográficas $e$ as demais que apresentaram valor de $p<0,20$ na análise bivariada foram incluídas no modelo de regressão de Poisson com variância robusta, com cálculos de Razões de Prevalência (RP) e seus respectivos intervalos de confiança (IC) de $95 \%$.

Para a análise de regressão, foi empregado um modelo hierárquico para determinação do agravo $^{15}$ (Figura 1), com as variáveis divididas em 3 blocos. No nível mais distal (Bloco 1) as variáveis foram as características demográficas (sexo e idade). No Bloco 2 foram analisadas as condições socioeconômicas, que hipoteticamente influenciam as questões relacionadas à saúde geral e bucal (Bloco 3), que foi o nível mais proximal do desfecho cárie radicular.

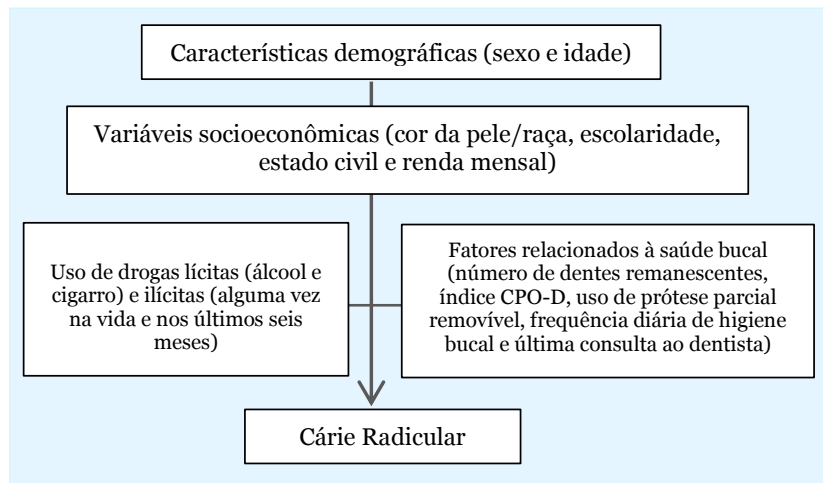

Figura 1. Modelo hierárquico para a investigação dos fatores associados à cárie radicular em indivíduos em situação de rua de Goiânia, GO. 
$\mathrm{Na}$ análise de regressão ajustada para os blocos superiores foram selecionadas as variáveis de cada bloco que tiveram significância estatística $(p<0,05)$ quando da análise de regressão múltipla interna a cada bloco. O projeto foi aprovado pelo Comitê de Ética em Pesquisa da UFG (Parecer consubstanciado, Protocolo n.045/2013). Os indivíduos que concordaram em participar assinaram um Termo de Consentimento Livre e Esclarecido.

RESULTADOS

Dos 116 indivíduos em situação de rua que participaram do estudo, 10 eram totalmente desdentados e foram excluídos da análise. As características sociodemográficas da amostra final $(\mathrm{N}=106)$ encontram-se na Tabela 1.

Tabela 1. Distribuição de frequência das características sociodemográficas da amostra e associação com a prevalência de cárie radicular.

\begin{tabular}{|c|c|c|c|c|}
\hline \multirow{2}{*}{$\begin{array}{c}\text { Características } \\
\text { sociodemográficas }\end{array}$} & \multirow{2}{*}{ Frequência } & \multicolumn{2}{|c|}{ Cárie radicular } & \multirow[b]{2}{*}{$\mathbf{p}$} \\
\hline & & Sim & Não & \\
\hline & $\begin{array}{l}\text { Mediana } \\
\text { (IIQ) }\end{array}$ & $\begin{array}{l}\text { Mediana } \\
\text { (IIQ) }\end{array}$ & $\begin{array}{l}\text { Mediana } \\
\text { (IIQ) }\end{array}$ & \\
\hline \multirow[t]{2}{*}{ Idade $(n=106)$} & $34,0(15,0)$ & $39,0(16)$ & $33,0(14)$ & $\mathbf{0 , 0 2 5}$ \\
\hline & n (\%) & $\%$ & $\%$ & \\
\hline \multicolumn{5}{|l|}{ Sexo } \\
\hline Feminino & $18(17,0)$ & 22,2 & 77,8 & \multirow{2}{*}{$0,658^{* *}$} \\
\hline Masculino & $88(83,0)$ & 27,3 & 72,7 & \\
\hline \multicolumn{5}{|l|}{ Cor da pele/raça $(\mathrm{n}=103)$} \\
\hline Branca & $22(21,4)$ & 22,7 & 77,3 & \multirow{2}{*}{$0,675^{* *}$} \\
\hline Não branca & $81(78,6)$ & 27,2 & 72,8 & \\
\hline \multicolumn{5}{|l|}{ Escolaridade $(\mathrm{n}=103)$} \\
\hline Alta (9 anos ou mais) & $35(34,0)$ & 22,9 & 77,1 & \multirow{3}{*}{$0,578^{* *}$} \\
\hline Baixa (até 8 anos) & $68(66,0)$ & 27,9 & 72,1 & \\
\hline \multicolumn{4}{|l|}{ Estado civil $(\mathrm{n}=103)$} & \\
\hline Casado/ União estável & $16(15,5)$ & 25,0 & 75,0 & \multirow[b]{2}{*}{$0,904^{* *}$} \\
\hline $\begin{array}{l}\text { Solteiro/Separado/ } \\
\text { Divorciado/Viúvo }\end{array}$ & $87(84,5)$ & 26,4 & 73,6 & \\
\hline \multicolumn{5}{|c|}{ Possui renda mensal $(\mathrm{n}=103)$} \\
\hline Sim & $67(65,0)$ & 20,9 & 79,1 & \multirow{2}{*}{$0,094^{* *}$} \\
\hline Não & $36(35,0)$ & 36,1 & 63,9 & \\
\hline \multicolumn{5}{|c|}{ Tempo em situação de rua $(\mathrm{n}=73)$} \\
\hline Até 1 ano & $42(57,5)$ & 21,4 & 78,6 & \multirow{2}{*}{$0,297^{* * *}$} \\
\hline Mais de 1 ano & $31(42,5)$ & 32,3 & 67,7 & \\
\hline
\end{tabular}

A idade variou de 18 a 68 anos (média= 36,3; mediana $=34,0$ ) e 96,2\% tinham menos de 60 anos. A maioria era do sexo masculino, tinha baixa escolaridade, era solteira/divorciada/viúva, possuía alguma renda mensal e se encontrava em situação de rua há 1 ano ou menos. Quanto à cor da pele/raça autodeclarada, 53,8\% eram pardos, $20,8 \%$ brancos, $17,0 \%$ negros, 3,8\% indígenas, 1,9\% amarelos, e três indivíduos $(2,8 \%)$ não informaram. Esta variável foi dicotomizada em brancos e não brancos para a análise estatística.

A prevalência do desfecho cárie radicular foi $26,4 \%(I C 95 \%=19,0-35,5)$. Todas as raízes afetadas estavam cariadas e nenhum dente apresentou raiz restaurada com ou sem cárie. $\mathrm{O}$ número de dentes com raízes cariadas e não tratadas por indivíduo variou de 0 a 8 (média= 0,76 ; $\mathrm{DP}=1,85$ ). A proporção de cárie radicular por indivíduo segundo o dente afetado nas duas arcadas encontra-se na Figura 2. $\mathrm{Na}$ arcada superior, o primeiro pré-molar direito (dente 14) apresentou a prevalência mais elevada $(6,6 \%)$. $\mathrm{Na}$ arcada inferior, os primeiros pré-molares (dentes 34 e 44) e o primeiro e segundo molares esquerdos (dentes 36 e 37 ) tiveram as maiores prevalências ( $3,8 \%$ cada). Os terceiros molares de ambas as arcadas (dentes 18, 28, 38 e 48) e os incisivos e caninos inferiores (dentes 33 a 43) tiveram as menores prevalências $(0,9 \%$ cada). O incisivo lateral superior direito (dente 22), o segundo pré-molar superior esquerdo (dente 25) e o segundo molar inferior direito (dente 47) não apresentaram cárie radicular.
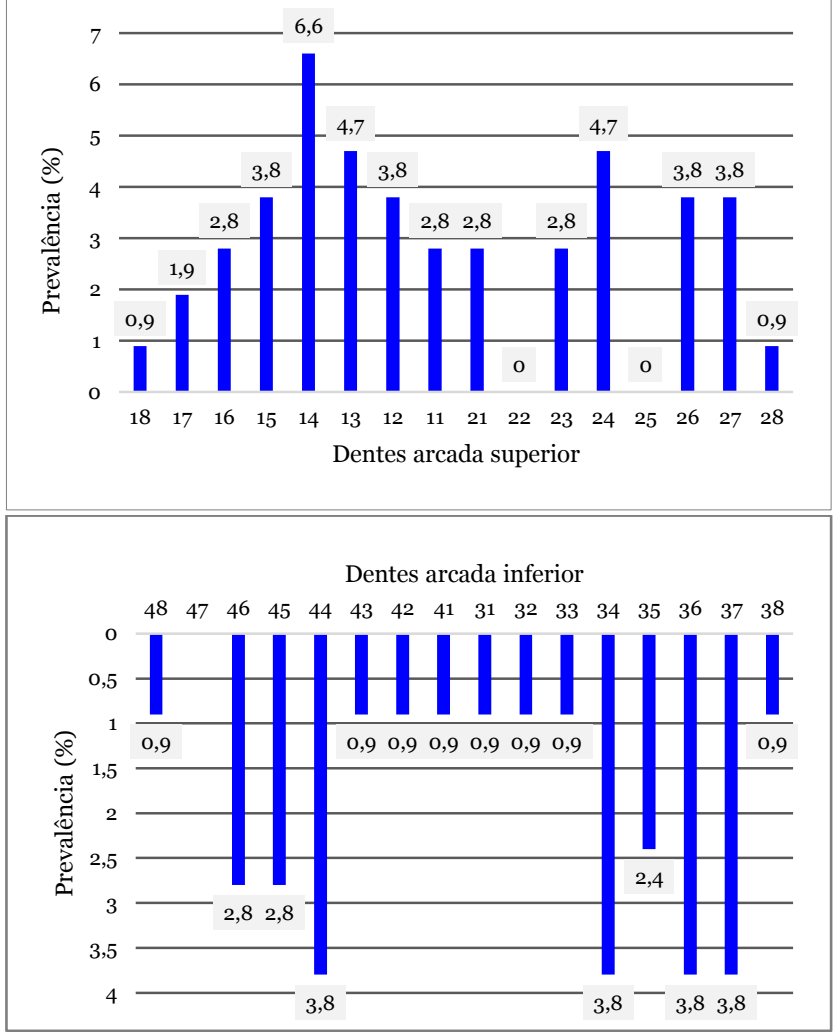

Figura 2. Prevalência de cárie radicular por indivíduo segundo o dente afetado nas arcadas superior e inferior.

A Tabela 2 apresenta a distribuição da amostra de acordo com as demais variáveis de saúde bucal e uso de drogas lícitas e ilícitas. O número de dentes remanescentes por indivíduo variou de 5 a 32 (média $=24,6$; mediana $=27,0$ ) e o índice CPO-D médio foi 12,8 (mediana= 12,0). A maior parte da amostra não usava prótese parcial removível, realizava higiene bucal 2 vezes ou mais ao dia e havia visitado o dentista há 3 anos ou mais. Quanto ao uso de drogas, a maioria relatou não ingestão de bebida alcoólica diariamente, consumo de cigarro atualmente, uso de substâncias ilícitas alguma vez e não uso destas nos seis meses anteriores à pesquisa.

As covariáveis que apresentaram associação com a presença de cárie radicular foram idade (Tabela 1), número de dentes remanescentes e índice CPO-D (Tabela 2). 
Maior proporção de indivíduos com cárie radicular foi encontrada nos grupos com idade, número de dentes remanescentes e índice CPO-D mais elevados $(p<0,05)$.

Os resultados da análise de regressão das covariáveis associadas com o desfecho prevalência de cárie radicular estão na Tabela 3. As variáveis dos Blocos 1 (idade), 2 (renda mensal) e 3 (número de dentes remanescentes e índice CPO-D) foram incluídas no modelo por apresentarem valor de $p<0,20$ na análise bivariada. $\mathrm{Na}$ etapa não ajustada, as variáveis idade, número de dentes remanescentes e índice CPO-D foram associadas ao desfecho.

Tabela 2. Distribuição de frequências das variáveis relacionadas à saúde bucal e uso de drogas e associação com a prevalência de cárie radicular.

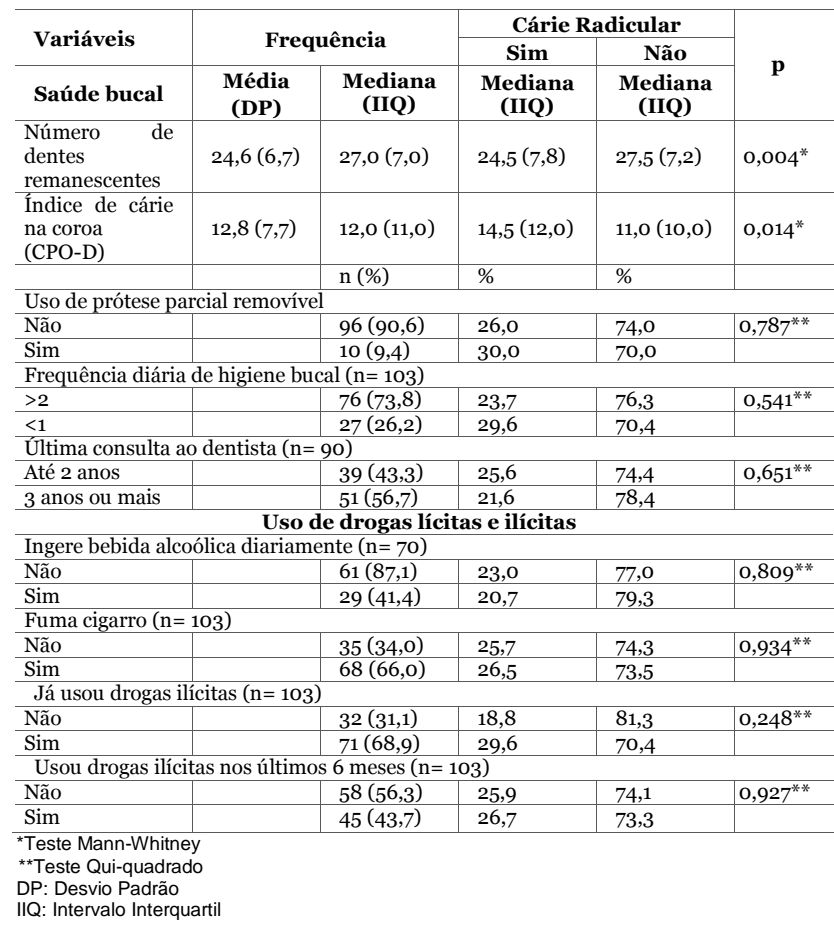

Tabela 3. Resultados da Regressão de Poisson da associação entre prevalência de cárie radicular e variáveis independentes

\begin{tabular}{|c|c|c|c|c|}
\hline \multirow{3}{*}{$\begin{array}{l}\text { Variáveis } \\
\text { independentes }\end{array}$} & \multicolumn{4}{|c|}{ Prevalência de cárie radicular } \\
\hline & \multicolumn{2}{|c|}{ Não ajustado } & \multicolumn{2}{|l|}{ Ajustado* $^{*}$} \\
\hline & RP (IC95\%) & $\mathbf{p}^{* *}$ & RP (IC95\%) & $\mathbf{p}^{* *}$ \\
\hline \multicolumn{5}{|l|}{ Bloco 1- Demográficas } \\
\hline Idade & $\begin{array}{l}1,03 \\
(1,00-1,05)\end{array}$ & 0,031 & $\begin{array}{l}1,03 \\
(1,00-1,05)\end{array}$ & $\mathbf{0 , 0 3 2}$ \\
\hline \multicolumn{5}{|l|}{ Sexo } \\
\hline Feminino & 1 & & 1 & \\
\hline Masculino & $\begin{array}{l}1,23 \\
(0,48-3,11)\end{array}$ & 0,666 & $\begin{array}{l}1,19 \\
(0,47-3,01)\end{array}$ & 0,719 \\
\hline \multicolumn{5}{|l|}{ Bloco 2-Socioeconômicas } \\
\hline \multicolumn{5}{|l|}{ Possui renda mensal } \\
\hline Sim & 1 & & & \\
\hline Não & $\begin{array}{l}1,73 \\
(0,91-3,27)\end{array}$ & 0,092 & $\begin{array}{l}1,44 \\
(0,75-2,79)\end{array}$ & 0,276 \\
\hline \multicolumn{5}{|c|}{ Bloco 3- Uso de drogas e saúde bucal } \\
\hline $\begin{array}{l}\text { Número de dentes } \\
\text { remanescentes }\end{array}$ & $\begin{array}{l}0,95 \\
(0,92-0,99)\end{array}$ & 0,006 & $\begin{array}{l}0,98 \\
(0,93-1,03)\end{array}$ & 0,386 \\
\hline $\begin{array}{l}\text { Indice de cárie na coroa } \\
\text { (CPO-D) }\end{array}$ & $\begin{array}{l}1,05 \\
(1,01-1,08)\end{array}$ & 0,006 & $\begin{array}{l}1,02 \\
(0,98-1,07)\end{array}$ & 0,310 \\
\hline \multicolumn{5}{|c|}{$\begin{array}{l}\text { *Ajustado pelas variáveis do mesmo bloco e acima } \\
\text { ** Teste de Wald } \\
\text { RP: Razão de Prevalência } \\
\text { IC95\%: Intervalo de Confiança de } 95 \%\end{array}$} \\
\hline
\end{tabular}

Após ajuste pelas variáveis do mesmo bloco e acima, apenas a idade dos participantes permaneceu significante $(p<0,05)$. Indivíduos mais velhos tiveram prevalência de cárie 3\% maior do que os indivíduos mais jovens $(\mathrm{RP}=1,03 ; \mathrm{IC} 95 \%=1,00-1,06)$.

DISCUSSÃO

O presente estudo buscou investigar a ocorrência e aspectos relacionados à cárie radicular entre PSR. Os resultados constituem o primeiro relato acerca dos fatores associados a esta condição neste grupo populacional e da prevalência em países em desenvolvimento. Mais de $1 / 4$ dos examinados $(26,4 \%)$ apresentaram dentes com experiência de cárie de raiz e chama a atenção o fato de todas as lesões estarem não tratadas, sem evidência de restaurações.

Comparações com estudos anteriores devem levar em consideração as diferenças metodológicas em relação às características da amostra e aos indicadores clínicos utilizados. Dois estudos foram realizados em PSR de cidades da Inglaterra. Entre 70 adultos e idosos predominantemente com problemas mentais, a prevalência (50\%) e a média de dentes afetados $(2,04)$ foram superiores às do presente estudo ${ }^{10}$. Dentre os 193 pacientes atendidos em um serviço de saúde para este grupo populacional entre 1992 e 2001, 8\% apesentaram raízes cariadas que necessitavam de tratamento, mas a prevalência incluindo raízes restauradas e outros dados descritivos não foram relatados ${ }^{11}$.

Resultados de uma revisão sistemática da literatura publicada até 2018 revelaram prevalência global de 41,5\% (IC95\%= 36,9$46,1)^{8}$. Estudos epidemiológicos sobre cárie radicular no Brasil são escassos. Prevalências mais baixas em comparação com a do presente estudo foram verificadas na população em geral de Goiânia e do país como um todo, de acordo com a Pesquisa Nacional de Saúde Bucal ${ }^{12}$, e um estudo no Estado de São Paulo ${ }^{16}$. Esta diferença era esperada, tendo em vista as precárias condições de vida e de acesso aos serviços de saúde neste grupo populacional, e confirmam a acentuada iniquidade na saúde quando se trata de populações vulneráveis.

Os dentes mais afetados (pré-molares e molares) estão em concordância com estudos anteriores ${ }^{9,17-19}$. Dentre os fatores investigados, somente a idade foi associada ao desfecho após ajuste por outras covariáveis. A associação encontrada, embora estatisticamente significante quando considerado $o$ valor de $p$, foi de baixa magnitude. No entanto, tem sido relatada em estudos anteriores na população em geral do Brasi $^{9,20}$ e de outros países ${ }^{18,19}$, e é considerada um preditor de cárie radicular ${ }^{7}$. A provável explicação é o maior tempo de exposição dos 
dentes aos determinantes da doença, assim como ocorre na cárie de coroa.

Os demais fatores que foram relacionados ao desfecho apenas nas comparações bivariadas (número de dentes remanescentes e índice de cárie na coroa), assim como a frequência de higiene bucal e o consumo de cigarro e álcool foram identificados em estudos anteriores ${ }^{6,7,18,21}$, mas parecem não ter relevância no contexto da população em situação de rua estudada. A relação entre o uso de drogas ilícitas e a cárie radicular em prisioneiros da cidade de Nova lorque foi relatada nos anos $1940^{22}$ e não tem sido investigada nos estudos mais recentes acerca da cárie radicular. Entre PSR, que geralmente apresentam alto consumo de drogas $^{23}$, esta relação era esperada.

Como limitação do presente estudo, destacamos o tamanho reduzido da amostra, que pode ter influenciado os resultados das associações investigadas. Além disso, a amostra de conveniência de indivíduos atendidos temporariamente em uma instituição não permite extrapolar os resultados para a população total de PSR do município estudado. Contudo, os estudos anteriores também foram realizados em instituições similares ou em serviços de saúde que atendem este grupo populacional $^{10,11}$. Esta opção se justifica pelas dificuldades de se realizar inquéritos de saúde nos locais onde estas pessoas vivem, que geralmente são dispersos e flutuantes. Ainda, os dentes com raízes expostas e sem experiência de cárie não foram registrados, impossibilitando o cálculo do Índice de Cárie de Raiz $^{24}$, o qual tem sido utilizado em estudos anteriores $\mathrm{s}^{9,17,19}$.

Os achados do presente estudo sugerem a necessidade de estratégias de saúde voltadas às especificidades deste grupo populacional que contribuam efetivamente para facilitar o acesso aos serviços odontológicos, atender as demandas existentes e prevenir problemas bucais na perspectiva da promoção da saúde geral e bucal.

CONCLUSÃO

Mais de $1 / 4$ dos indivíduos em situação de rua pesquisados apresentaram cárie radicular e a prevalência foi associada ao aumento da idade. A inexistência de raízes restauradas sugere a necessidade de promover o acesso deste grupo populacional aos serviços de saúde.

\section{AGRADECIMENTOS}

Os autores agradecem as seguintes fontes de financiamento: Coordenação de
Aperfeiçoamento de Pessoal de Nível Superior (CAPES) - Código 001, e Escritório das Nações Unidas sobre Drogas e Crime (UNODC - CartaAcordo 100/2013), em parceria com o Ministério da Saúde - Coordenação de DST/HIV/Aids e hepatites virais - edital: 003/2013.

REFERÊNCIAS

1. Brasil. Decreto Presidencial no 7.053, de 23 de dezembro de 2009. Institui a Política Nacional para a População em Situação de Rua e seu comitê intersetorial de acompanhamento e monitoramento. Diário Oficial da União 2009; $24 \mathrm{dez}$.

2. Brasil. Meta Instituto de Pesquisa de Opinião/Ministério do Desenvolvimento Social e de Combate à Fome. Secretaria de Avaliação e Gestão da Informação. Pesquisa Nacional sobre a População em Situação de Rua. Sumário executivo. Brasília: Ministério do Desenvolvimento Social e de Combate à Fome; 2008.

3. Natalino MAC. Estimativa da população em situação de rua no Brasil. Instituto de Pesquisa Econômica Aplicada. Brasília, DF: Rio de Janeiro: Ipea, 2016.

4. Brasil. Ministério da Saúde. Secretaria de Atenção à Saúde. Departamento de Atenção Básica. Manual sobre o cuidado à saúde junto a população em situação de rua. Brasília: Ministério da Saúde; 2012.

5. Brasil. Ministério da Saúde. Secretaria de Gestão Estratégica e Participativa. Departamento de Apoio à Gestão Participativa. Saúde da população em situação de rua: um direito humano. Brasília: Ministério da Saúde; 2014.

6. Ritter AV, Shugars DA, Bader JD. Root caries risk indicators: a systematic review of risk models. Community Dent Oral Epidemiol. 2010;38:383-97.

7. Zhang J, Leung KCM, Sardana D, Wong MCM, Lo ECM. Risk predictors of dental root caries: A systematic review. J Dent. 2019;89:103166.

8. Pentapati KC, Siddiq H, Yeturu SK. Global and regional estimates of the prevalence of root caries - Systematic review and meta-analysis. Saudi Dent J. 2019;31:3-15.

9. Marques RAA, Antunes JLF, Sousa MLR, Peres MA, Frazão P. Prevalência e extensão da cárie dentária radicular em adultos e idosos brasileiros. Rev Saúde Pública. 2013;47(Supl 3):59-68.

10. Waplington J, Morris J, Bradnock G. The dental needs, demands and attitudes of a group of homeless people with mental health problems. Community Dent Health. 2000;17:134-7.

11. Daly B, Newton JT, Batchelor P. Patterns of dental service use among homeless people using a targeted service. J Public Health Dent. 2010;70:45-51. 
12. Brasil. Ministério da Saúde. Secretaria de Atenção à Saúde. Departamento de Atenção Básica. Projeto SB Brasil 2010: Pesquisa Nacional de Saúde Bucal. Resultados principais. Brasília: Ministério da Saúde; 2011.

13. World Health Organization. Oral health surveys: basic methods. 4. ed. Geneva; 1997.

14. Instituto Brasileiro de Geografia e Estatística (IBGE). Censo Brasileiro 2010. Rio de Janeiro: IBGE; 2010.

15. Victora CG, Huttly SR, Fuchs SC, Olinto MTA. The role of conceptual frameworks in epidemiological analysis: a hierarchical approach. Int J Epidemiol. 1997;26:224-7.

16. Rihs LB, Sousa MLR, Wada RS. Prevalência de cárie radicular em adultos e idosos na região sudeste do Estado de São Paulo, Brasil. Cad Saúde Pública. 2005;21:311-6.

17. Splieth Ch, Schwahn Ch, Bernhardt O, John U. Prevalence and distribution of root caries in Pomerania, North-East Germany. Caries Res. 2004;38:333-40.

18. Kularatne S, Ekanayake L. Root surface caries in older individuals from Sri Lanka. Caries Res. 2007;41:252-6.

19. Du MQ, Jiang H, Tai BJ, Zhou Y, Wu B, Bian Z. Root caries patterns and risk factors of middleaged and elderly people in China. Community Dent Oral Epidemiol. 2009;37:260-6.

20. Bidinotto $A B$, Martins $A B$, Dos Santos $C M$, Hugo FN, Hilgert JB, Celeste RK, Padilha DM, De Marchi RJ. Four-year incidence rate and predictors of root caries among communitydwelling south Brazilian older adults. Community Dent Oral Epidemiol. 2018;46: 125-31.

21. Nicolau B, Srisilapan P, Marcenes W. Number of teeth and risk of root caries. Gerodontology. 2000;17:91-6.

22. Hecht SS, Friedman J. High incidence of cervical dental caries among drug addicts. Oral Surg. 1949;2:1428-42.

23. Fazel S, Khosla V, Doll H, Geddes J. The prevalence of mental disorders among the homeless in western countries: systematic review and meta-regression analysis. PLoS Med. 2008;5:e225.

24. Katz RV. Assessing root caries in populations: the evolution of the root caries index. J Public Health Dent. 1980;40:7-16.

\section{CONFLITO DE INTERESSES}

Os autores declaram não haver conflitos de interesse

AUTOR PARA CORRESPONDÊNCIA

\section{Profa. Maria do Carmo Matias Freire}

Faculdade de Odontologia,

Universidade Federal de Goiás (UFG)

Praça Universitária $\mathrm{s} / \mathrm{n}$, Setor Universitário

74605-220 Goiânia-GO, Brasil

mcmfreire@yahoo.com.br 